\title{
Expression of multidrug resistance-associated proteins and their relation to postoperative individualized chemotherapy in gastric cancer
}

Pengfei Yu*, Yian Du, Xiangdong Cheng, Qiming Yu, Ling Huang and Ruizeng Dong

\begin{abstract}
Background: Adjuvant chemotherapy could reduce residual tumor cells and prevent relapse, however, not all patients are suitable for adjuvant chemotherapy. Screening appropriate patients based on molecular markers for individualized adjuvant chemotherapy is necessary.

Methods: Between June 2002 and June 2004, 119 patients who underwent radical gastrectomy were retrospectively analyzed. Some patients had adjuvant chemotherapy based on platinum and 5-FU for four to six cycles. Topoisomerase II (ToPo II) negative, multidrug resistance protein (MRP) positive and glutathione S-transferase

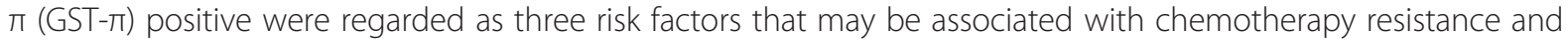
poor prognosis. Patients were divided into two groups: a high-risk group ( $\geq 2$ risk factors) and a low-risk group ( $<2$ risk factors), and tumor recurrence and patient survival time of the two groups were analyzed.
\end{abstract}

Results: The average recurrence time of the low-risk group was significantly longer than that of the high-risk group $(21.29 \pm 11.10$ versus $15.16 \pm 8.05$ months, $P<0.01)$. The 3 -year and 5 -year survival rates of the high-risk group were $57.4 \%$ and $42.6 \%$, however, it had no significant difference compared to $66.2 \%$ and $58.5 \%$ of the low-risk group $(P>0.05)$. In the high-risk group, the 3-year survival rates of patients with/without chemotherapy were $62.1 \%$ and $52.0 \%$ and the 5 -year survival rates were $44.8 \%$ and $40.0 \%$, respectively, but the difference was not statistically significant $(P>0.05)$. In the low-risk group, the 3 -year survival rates of patients with/without chemotherapy were $81.2 \%$ and $51.5 \%$, and the 5 -year survival rates were $71.9 \%$ and $45.5 \%$, respectively, these differences were statistically significant $(P<0.05)$.

Conclusions: Combined detection of the multidrug resistance (MDR)-related proteins ToPo II, MRP and GST-r may be prospectively valuable for postoperative individualized chemotherapy and in further predicting the outcomes of gastric cancer patients.

Keywords: stomach neoplasms, multidrug resistance-associated proteins, chemotherapy, prognosis

\section{Background}

There is still a high risk of recurrence and metastasis after radical gastric surgery, and adjuvant chemotherapy may reduce postoperative residual tumor cells and prevent relapse $[1,2]$. In recent years, new results of randomized controlled studies indicate that postoperative chemotherapy can improve the prognosis of the patients $[3,4]$. These results have been accepted in the NCCN Gastric Cancer Clinical Practice Guidelines and are

\footnotetext{
*Correspondence: yupengfei23@163.com

Department of Abdominal Surgery, Zhejiang Cancer Hospital, 38\# Guangji Road, Hangzhou 310022, China
}

recommended as the basis of postoperative treatment programs. However, some issues still need to be addressed: (1) subgroup analysis indicates that some patients (female, node-negative, late stage, older, etcetera) do not benefit from adjuvant therapy and (2) problems occur with chemotherapy toxicity and compliance, which causes some patients to withdraw from treatment because of adverse events.

So, postoperative adjuvant chemotherapy is beneficial for some patients, however, it may increase the treatment burden and reduce the immunity of other patients. Therefore, it is too early to determine a program as 
standard adjuvant chemotherapy for gastric cancer. There are still many issues that need high-quality research to answer before individualized adjuvant chemotherapy becomes standard. What is particularly worth mentioning is that the ToGA study has confirmed the value of Herceptin in the treatment of advanced gastric cancer [5]. Recently, Deng and colleagues provided for the first time a detailed molecular map of genomic alterations in gastric cancer, which revealed several promising targets for subtype-specific therapies [6]. Screening appropriate patients based on molecular markers will become a major research direction for individualized chemotherapy [7].

Multidrug resistance-associated proteins topoisomerase II (ToPo II), multidrug resistance protein (MRP) and glutathione S-transferase $\pi$ (GST- $\pi$ ) are the basis of multidrug resistance in malignant tumors [8,9]. It had been confirmed that MRP and GST- $\pi$ overexpression, and decreased expression of ToPo II are important mechanisms mediating multidrug resistance [10]. Therefore, we carried out this study of multidrug resistance (MDR) gene-associated proteins in postoperative individualized treatment for gastric cancer.

\section{Methods}

\section{Patients and tissue samples}

Between June 2002 and June 2004, a total of 119 patients who underwent radical gastrectomy at the Department of Abdominal Surgery, Zhejiang Cancer Hospital, were retrospectively analyzed. Of these patients, 77 cases were males and 42 cases were females, ages 25 to 78 years (mean $57.3 \pm 6.7$ years). Phase I/II included 39 cases and Phase III/IV included 80 cases; lesions $\geq 5 \mathrm{~cm}$ were found in 76 cases and lesions $<5 \mathrm{~cm}$ were found in 43 cases; and patients with/without lymph nodes metastases were identified for 93 cases and 26 cases, respectively. None of the patients received preoperative chemotherapy or other treatment for the tumor, and some patients had adjuvant chemotherapy based on platinum and 5flurouracil (5-FU) for four to six cycles. Written informed consent was obtained from all the study participants. The study was approved by the Ethics Committee of Zhe Jiang Cancer Hospital.

\section{Immunohistochemical staining}

The antibodies used in this study were purchased from GBI Company (Golden Bridge International, Inc., Mukilteo, WA, USA). Immunohistochemical staining was carried out on the formalin-fixed, 4- $\mu$ m-thick, paraffin-embedded tissue specimens. Pancreas, colon, and ovary samples were used as positive controls for Topo II, MRP, and GST- $\pi$, respectively. The specimens were evaluated independently by two pathologists in a blind fashion. Only cells with brown-colored staining were considered as positive. The intensity of expression of $M D R$-related proteins was stratified into four categories that were scored as follows: 1) negative (-) had no appreciable cytomembrane, nuclear or cytoplasmic staining or had staining in $<10 \%$ of neoplastic cells; 2) $1+$ had appreciable staining in 10 to $25 \%$ of neoplastic cells; 3 ) $2+$ had appreciable staining in 25 to $75 \%$ of neoplastic cells; and 4) $3+$ had appreciable staining in $>75 \%$ of neoplastic cells.

\section{Patient follow-up}

Patients received routine follow-up after radical gastrectomy once every quarter for two years and thereafter, once every half year (patients who received chemotherapy were followed up with chemotherapy cycles). ToPo II negative, MRP positive and GST- $\pi$ positive were regarded as three risk factors that may be associated with chemotherapy resistance and poor prognosis. Patients were divided into two groups: the high-risk group ( $\geq 2$ risk factors) and the low-risk group ( $<2$ risk factors), and the tumor recurrence and patient survival time of the two groups were analyzed.

\section{Statistical analysis}

All the experiment data were integrated into a comprehensive data set. Numerical data were recorded directly and measurement data were described as median and range. Statistical analysis was performed on SPSS software version 16.0 (SPSS Inc. Chicago, IL), and $P<0.05$ was considered as statistically significant.

\section{Results}

The positive staining of ToPo II was recognized to be expressed in the cell nucleus (Figure 1A), whereas MRP and GST- $\pi$ were expressed in the cytoplasm of malignant cells (Figure 1B and Figure 1C).

The expression rate of ToPo II in normal tissues (75.1\%) was higher than that in tumor tissues (73.9\%) (not significant, $P>0.05$ ). When comparing the well-, moderately and poorly differentiated degree of expression, a significant correlation was shown between ToPo II expression and the level of differentiation (86.3\%, $64.5 \%$ and $64.9 \%$, respectively, $P<0.05$ ). As for MRP, the positive rate was $42.9 \%$ in tumor tissues whereas all the normal gastric tissues were not stained. No significant differences in MRP expression were found in relation to the clinicopathological factors. The positive rate of GST$\pi$ in patients with gastric cancer was higher than that of the normal tissues $(51.3 \%$ versus $23.2 \%, P<0.05)$. Significant differences in GST- $\pi$ expression were also found in relation to sex (male versus female, 59.7\% versus $35.7 \%, P<0.05$ ) and differentiation (well, moderately and poorly, $40.5 \%, 41.9 \%$, and $64.7 \%$, respectively, $P<0.05)$ (Table 1). 

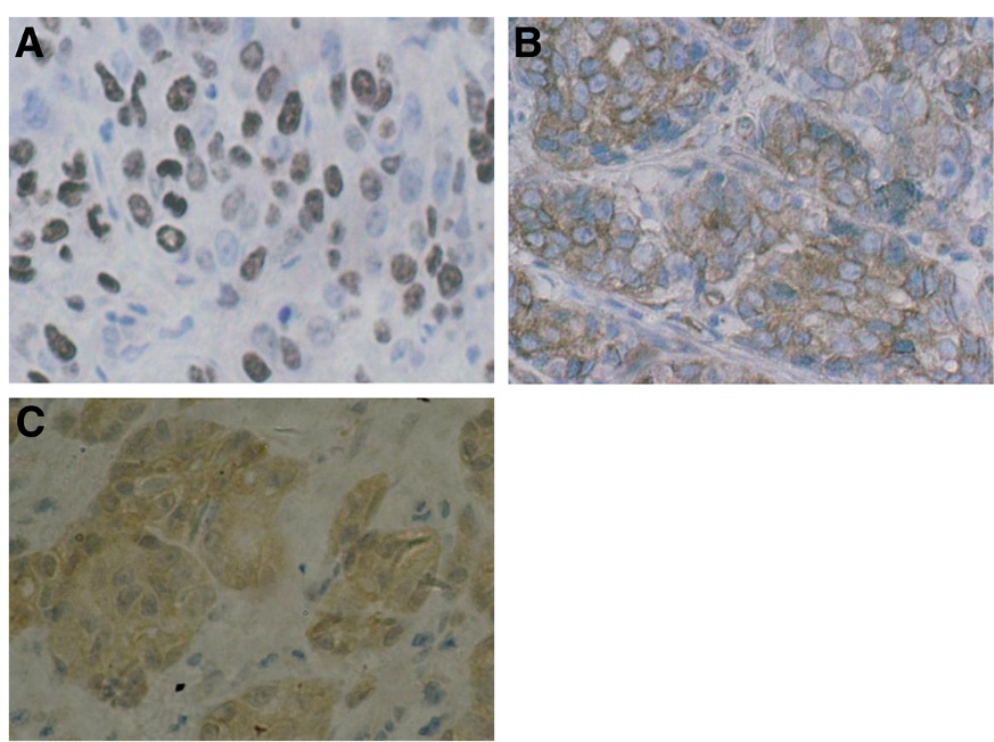

Figure 1 Immunohistochemical staining of multidrug resistance-associated proteins. A Immunohistochemical staining of ToPo II was identified in the cell nucleus (original magnification $\times 400$ ). B Immunohistochemical staining of MRP was recognized to be expressed in the cytoplasm of malignant cells (original magnification $\times 400$ ). C Immunohistochemical staining of GST- $\pi$ was recognized to be expressed in the cytoplasm of malignant cells (original magnification $\times 400$ ).

Table 1 The expression of ToPo II,MRP, and GST- $\pi$ and their relationship with clinicopathological factors

\begin{tabular}{|c|c|c|c|c|c|c|c|c|c|c|}
\hline \multirow{2}{*}{$\begin{array}{l}\text { Clinicopathologic } \\
\text { Features }\end{array}$} & \multirow[t]{2}{*}{ Cases } & \multicolumn{3}{|l|}{ ToPo II } & \multicolumn{3}{|l|}{ MRP } & \multicolumn{3}{|l|}{ GST-ח } \\
\hline & & $+(\%)$ & $x^{2}$ & $P$ & $+(\%)$ & $x^{2}$ & $P$ & $+(\%)$ & $x^{2}$ & $P$ \\
\hline \multicolumn{11}{|l|}{ Sex } \\
\hline Male & 77 & $54(70.1 \%)$ & & & 29(37.7\%) & & & $46(59.7 \%)$ & & \\
\hline Female & 42 & $34(81.0 \%)$ & 1.65 & 0.198 & $22(52.4 \%)$ & 2.40 & 0.121 & $15(35.7 \%)$ & 6.27 & $0.012^{*}$ \\
\hline \multicolumn{11}{|l|}{ Age } \\
\hline$\leq 50 y$ & 33 & $24(72.7 \%)$ & & & $16(48.5 \%)$ & & & $14(42.4 \%)$ & & \\
\hline$>50 y$ & 86 & $64(74.4 \%)$ & 0.03 & 0.851 & $35(40.7 \%)$ & 0.59 & 0.442 & $47(54.7 \%)$ & 1.42 & 0.232 \\
\hline \multicolumn{11}{|l|}{ Tumor size } \\
\hline$\geq 5 \mathrm{~cm}$ & 76 & $59(77.6 \%)$ & & & $35(46.1 \%)$ & & & $40(52.6 \%)$ & & \\
\hline$<5 \mathrm{~cm}$ & 43 & $29(67.4 \%)$ & 1.48 & 0.224 & $16(37.2 \%)$ & 0.88 & 0.349 & $21(48.8 \%)$ & 0.16 & 0.691 \\
\hline \multicolumn{11}{|l|}{ Differentiation } \\
\hline Well & 51 & $44(86.3 \%)$ & & & $20(39.2 \%)$ & & & $33(40.5 \%)$ & & \\
\hline Moderately & 31 & $20(64.5 \%)$ & & & $13(41.9 \%)$ & & & $13(41.9 \%)$ & & \\
\hline Poorly & 37 & $24(64.9 \%)$ & 7.04 & $0.029^{*}$ & 18(48.6\%) & 0.79 & 0.672 & $15(64.7 \%)$ & 6.47 & $0.039 *$ \\
\hline \multicolumn{11}{|l|}{ TNM staging } \\
\hline$|/| \mid$ & 39 & $25(64.1 \%)$ & & & 17 (43.6\%) & & & $16(41.0 \%)$ & & \\
\hline III/IV & 80 & $63(78.8 \%)$ & 2.92 & 0.087 & $34(42.5 \%)$ & 2.57 & 0.109 & $45(56.3 \%)$ & 2.43 & 0.119 \\
\hline \multicolumn{11}{|l|}{ Lymph node } \\
\hline Positive & 93 & $71(76.3 \%)$ & & & $40(43.0 \%)$ & & & $47(50.5 \%)$ & & \\
\hline Negative & 26 & $17(65.4 \%)$ & 1.27 & 0.260 & $11(42.3 \%)$ & 0.004 & 0.949 & $14(53.8 \%)$ & 0.09 & 0.765 \\
\hline
\end{tabular}




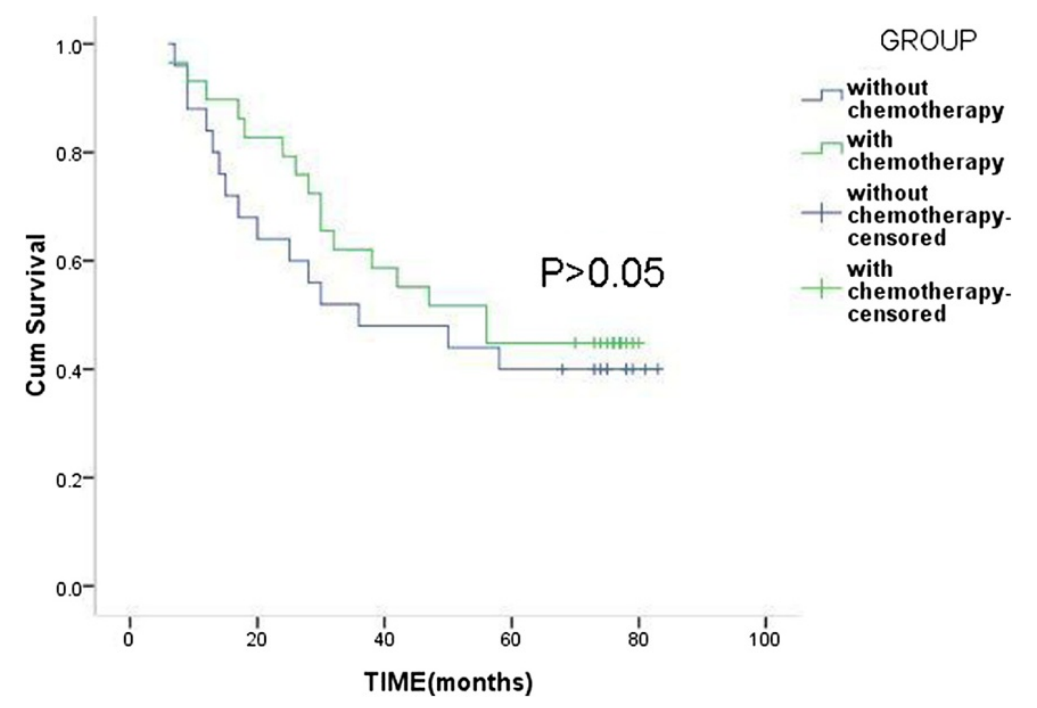

Figure 2 Overall survival of patients with or without chemotherapy in the high-risk group.

The 3- and 5-year survival rates of the 119 patients were $57.3 \%$ and $49.2 \%$, respectively. No statistical difference was observed between single protein (ToPo II、MRP or GST- $\pi$ ) expression and the recurrence or survival time. When patients were divided into two groups: the high-risk group ( $\geq 2$ risk factors) and the low-risk group ( $<2$ risk factors), the average recurrence time of the low-risk group was $21.29 \pm 11.10$ months and was significantly longer than $15.16 \pm 8.05$ months of the high-risk group $(P<0.01)$. The 3 -year and 5 -year survival rate of the high-risk group was $57.4 \%$ and 42.6\%; however, there was no significant difference compared to the $66.2 \%$ and $58.5 \%$ of the low-risk group $(P>0.05)$.
In the high-risk group, the 3-year survival rates of patients with chemotherapy and patients without chemotherapy were $62.1 \%$ and $52.0 \%$, and the 5 -year survival rates were $44.8 \%$ and $40.0 \%$, but the difference was not statistically significant $(P>0.05)$ (Figure 2$)$. In the lowrisk group, the 3-year survival rates of patients with chemotherapy and patients without chemotherapy were $81.2 \%$ and $51.5 \%$, the 5 -year survival rates were $71.9 \%$ and $45.5 \%$, and the difference was statistically significant $(P<0.05)$ (Figure 3).

\section{Discussion}

Adjuvant chemotherapy after operation has been considered as necessary to eliminate systemic micrometastases

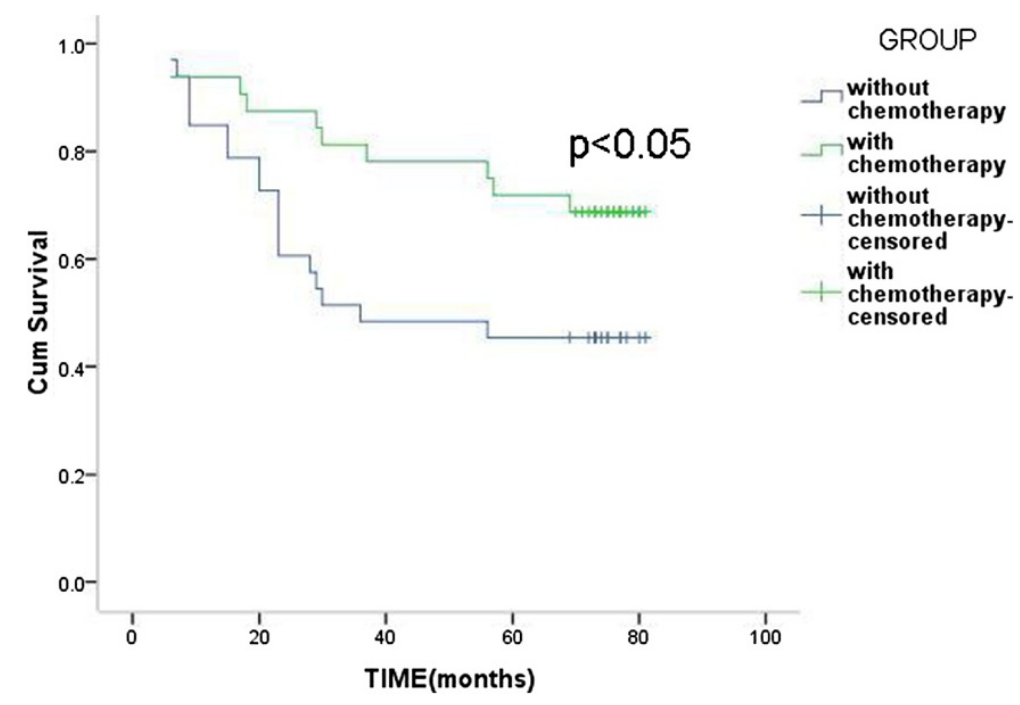

Figure 3 Overall Survival of patients with or without chemotherapy in the low-risk group. 
and remnant malignant cells to the fullest extent possible, ultimately improving survival [11,12]. Unfortunately, so far, this kind of adjuvant treatment strategy has been disappointing as a result of multidrug resistance (MDR) of malignant cells to different chemotherapeutic agents $[13,14]$. Therefore, detection and evaluation of $M D R$ genes or proteins may help guide adjuvant chemotherapy in gastric cancer and determine the prognosis of patients.

MRP, one of the most studied mechanisms of MDR, acts as an ATP-dependent outward transport pump and decreases intracellular accumulation of drugs by reducing the co-transport mechanism of glutathione [15]. Several previous studies have also indicated that overexpression of MRP most frequently predicts MDR. MRP confers resistance to alkylating agents, cyclophosphamide and other drugs [16]. GST- $\pi$ is a multifunctional enzyme that plays a critical role in cellular detoxification by catalyzing the conjugation of reduced glutathione to hydrophobic and electrophilic compounds [17]. GST- $\pi$ is considered to be associated with the efflux of cisdiaminodichloroplatin (CDDP), flurouracil and doxorubicin (DOX) through ATP-binding cassette transporters [18]. ToPo II is the target of several anticancer agents, such as doxorubicin, VM26, VP16 and mitoxantrone [19]. The decreased expression of ToPo II and changes in enzyme activity result in the dissociation of cleavable complexes and reduced DNA damage, and finally cause the drug resistance [20].

In our study, statistical analysis indicates that none of the three proteins were significantly correlated with the recurrence and survival rates, so the determination of a single indicator of the effectiveness of adjuvant chemotherapy is difficult. Because ToPo II negative, MRP positive and GST- $\pi$ positive were regarded as three risk factors that may be associated with chemotherapy resistance and poor prognosis, these patients were divided into two groups: the high-risk group ( $\geq 2$ risk factors) and the low-risk group ( $<2$ risk factors). The recurrence time of the low-risk group was significantly longer than that of the high-risk group, suggesting that the decreased expression of ToPo II and high expression of MRP and GST- $\pi$ was associated with tumor invasion, recurrence and poor prognosis, and this conclusion had been confirmed in ovarian cancer [21]. In the low-risk group, the 3-year and 5-year survival rate of patients with chemotherapy was higher than that of the patients without chemotherapy. This result indicated that 5-Fu and platinum-based postoperative chemotherapy can increase survival benefits for patients in the low-risk group. Chemotherapy resistance was rare in these patients, and in theory, postoperative chemotherapy can be done fully fit, and the prognosis of the patient will be significantly improved. In the high-risk group, the 3-year and 5-year survival rate of patients with chemotherapy was higher than that of the patients without chemotherapy, but the difference was not statistically significant. Therefore, the 5-Fu and platinum-based adjuvant chemotherapy did not improve the prognosis of the high-risk group, and for such patients, postoperative chemotherapy needs to be carefully discussed and selected. These conclusions were based on a small number of cases and may have some limitations. A large sample of patients is being followed up in our center, and detailed results, including the subgroup analysis (gender, lymph node metastasis, staging, etcetera.), will be reported in the near future.

\section{Conclusions}

Therefore, combined determination of MDR-related proteins ToPo II, MRP and GST- $\pi$ may be prospectively valuable for optimizing chemotherapy regimes and further predicting the outcomes of patients. Further research should focus on the combined detection of molecular markers (such as HER-2, MDR-related proteins, RTK/RAS signaling molecules, etcetera.) for individualized chemotherapy and carry out multicenter clinical trials, the results may be exciting.

\section{Consent}

Written informed consent was obtained from the patient for the publication of this report and any accompanying images.

\section{Abbreviations}

CDDP: CIS-diaminodichloroplatin; DOX: doxorubicin; GST-r: glutathione S-transferase $\pi$; MDR: multidrug resistance; MRP: multidrug resistance protein; ToPo II: topoisomerase II; 5-FU: 5-flurouracil.

\section{Competing interests}

No benefits in any form have been received or will be received from a commercial party related directly or indirectly to the subject of this article. The authors declare that they have no competing interests.

\section{Authors' contributions}

Yu PF, Du YA, Cheng XD, Yu QM, Huang L, Dong RZ designed and conducted the study, analyzed the data, and helped to write the manuscript. Yu PF is the principal investigator, and revised and edited the manuscript. All authors read and approved the final manuscript.

\section{Acknowledgements}

This study was supported by Natural Science Foundation of Zhejiang Province of China (No.LY14H160007).

Received: 12 February 2014 Accepted: 25 September 2014 Published: 11 October 2014

\section{References}

1. Kodera Y, Ito S, Mochizuki Y, Kondo K, Koshikawa K, Suzuki N, Kojima H, Kojima T, Matsui T, Takase T, Tsuboi K, Fujiwara M, Nakao A: A phase II study of radical surgery followed by postoperative chemotherapy with S-1 for gastric carcinoma with free cancer cells in the peritoneal cavity (CCOG0301 study). Eur J Surg Oncol 2009, 35:1158-1163.

2. Omura T, Shimada Y, Nagata T, Okumura T, Fukuoka J, Yamagishi F, Taijka S, Nakajima S, Kawabe A, Tsukada K: Relapse-associated microRNA in gastric cancer patients after S-1 adjuvant chemotherapy. Oncol Rep 2014, 31:613-618. 
3. Bang YJ, Kim YW, Yang HK, Chung HC, Park YK, Lee KH, Lee KW, Kim YH, Noh SI, Cho JY, Mok YJ, Kim YH, Ji J, Yeh TS, Button P, Sirzén F: Noh SH; CLASSIC trial investigators: Adjuvant capecitabine and oxaliplatin for gastric cancer after D2 gastrectomy (CLASSIC): a phase 3 open-label, randomised controlled trial. Lancet 2012, 379:315-321.

4. Sasako M, Sakuramoto $S$, Katai H, Kinoshita T, Furukawa H, Yamaguchi T, Nashimoto A, Fujii M, Nakajima T, Ohashi Y: Five-year outcomes of a randomized phase III trial comparing adjuvant chemotherapy with S-1 versus surgery alone in stage II or III gastric cancer. J Clin Oncol 2012, 29:4387-4393.

5. Bang YJ, Van Cutsem E, Feyereislova A, Chung HC, Shen L, Sawaki A, Lordick F, Ohtsu A, Omuro Y, Satoh T, Aprile G, Kulikov E, Hill J, Lehle M, Rüschoff J: Kang YK; ToGA Trial Investigators: Trastuzumab in combination with chemotherapy versus chemotherapy alone for treatment of HER2positive advanced gastric or gastro-oesophageal junction cancer (ToGA): a phase 3, open-label, randomised controlled trial. Lancet 2012, 376:687-697.

6. Deng N, Goh LK, Wang H, Das K, Tao J, Tan IB, Zhang S, Lee M, Wu J, Lim KH, Lei Z, Goh G, Lim QY, Tan AL, Sin Poh DY, Riahi S, Bell S, Shi MM, Linnartz R, Zhu F, Yeoh KG, Toh HC, Yong WP, Cheong HC, Rha SY, Boussioutas A, Grabsch H, Rozen S, Tan P: A comprehensive survey of genomic alterations in gastric cancer reveals systematic patterns of molecular exclusivity and co-occurrence among distinct therapeutic targets. Gut 2012, 61:673-684.

7. Liu Y, Chen J, Shao N, Feng Y, Wang Y, Zhang L: Clinical value of hematologic test in predicting tumor response to neoadjuvant chemotherapy with esophageal squamous cell carcinoma. World J Surg Oncol 2014, 12:43.

8. Lu D, Shi HC, Wang ZX, Gu XW, Zeng YJ: Multidrug resistance-associated biomarkers PGP, GST-pi, Topo-II and LRP as prognostic factors in primary ovarian carcinoma. Br J Biomed Sci 2011, 68:69-74.

9. Kunjachan S, Rychlik B, Storm G, Kiessling F, Lammers T: Multidrug resistance: Physiological principles and nanomedical solutions. Adv Drug Deliv Rev 2013, 65:1852-1865.

10. Shi H, Lu D, Shu Y, Shi W, Lu S, Wang K: Expression of multidrug resistance-related proteins $\mathrm{P}$-glycoproteinglutathione-s-transferases, ToPoisomerase-II and Lung resistance protein in primary gastric cardiac adenocarcinoma. Cancer Invest 2008, 26:344-351.

11. Wu Y, Wei ZW, He YL, Schwarz RE, Smith DD, Xia GK, Zhang CH: Efficacy of adjuvant XELOX and FOLFOX6 chemotherapy after D2 dissection for gastric cancer. World J Gastroenterol 2013, 19:3309-3315.

12. Fujitani K: Overview of adjuvant and neoadjuvant therapy for resectable gastric cancer in the East. Dig Surg 2013, 30:119-129.

13. Li Y, Tan BB, Zhao Q, Fan LQ, Liu Y, Hao YJ, Zhao XF: Tumor chemosensitivity is correlated with expression of multidrug resistance associated factors in variously differentiated gastric carcinoma tissues. Hepatogastroenterology 2013, 60:213-216.

14. Zhu CY, LV YP, Yan DF, Gao FL: Knockdown of MDR1 Increases the Sensitivity to Adriamycin in Drug Resistant Gastric Cancer Cells. Asian Pac J Cancer Prev 2013, 14:6757-6760

15. Xu HW, Xu L, Hao JH, Qin CY, Liu H: Expression of P-glycoprotein and multidrug resistance-associated protein is associated with multidrug resistance in gastric cancer. $J$ Int Med Res 2010, 38:34-42.

16. Ge J, Chen Z, Wu S, Chen J, Li X, Li J, Yin J, Chen Z: Expression levels of insulin-like growth factor-1 and multidrug resistance-associated protein-1 indicate poor prognosis in patients with gastric cancer. Digestion 2009, 80:148-158.

17. Soh Y, Goto S, Kitajima M, Moriyama S, Kotera K, Nakayama T, Nakajima H Kondo T, Ishimaru T: Nuclear localisation of glutathione $\mathrm{S}$-transferase pi is an evaluation factor for drug resistance in gynaecological cancers. Clin Oncol (R Coll Radiol) 2005, 17:264-270.

18. Gate L, Majumdar RS, Lunk A, Tew KD: Influence of glutathione S-transferase pi and p53 expression on tumor frequency and spectrum in mice. Int $J$ Cancer 2005, 113:29-35.

19. Chen W, Qiu J, Shen YM: Topoisomerase Ila, rather than $\Perp \beta$, is a promising target in development of anti-cancer drugs. Drug Discov Ther 2012, 6:230-237.
20. Chau M, Christensen JL, Ajami AM, Capizzi RL: Amonafide, a topoisomerase II inhibitor, is unaffected by P-glycoprotein-mediated efflux. Leuk Res 2008, 32:465-473.

21. Cao DY, Shen K, Yang JX, Guan J: The expression of MRP, GST-pi, Topo Ilalpha and COX-2 in epithelial ovarian cancer and its relationship to drug resistance and prognosis. Zhonghua Yi Xue Za Zhi 2007, 87:1738-1741.

doi:10.1186/1477-7819-12-307

Cite this article as: Yu et al.: Expression of multidrug resistanceassociated proteins and their relation to postoperative individualized chemotherapy in gastric cancer. World Journal of Surgical Oncology 2014 12:307.

\section{Submit your next manuscript to BioMed Central and take full advantage of:}

- Convenient online submission

- Thorough peer review

- No space constraints or color figure charges

- Immediate publication on acceptance

- Inclusion in PubMed, CAS, Scopus and Google Scholar

- Research which is freely available for redistribution

Submit your manuscript at www.biomedcentral.com/submit
C BioMed Central 